Personality and Mental Health

9: 84-86 (2015)

Published online 21 January 2015 in Wiley Online Library

(wileyonlinelibrary.com) DOI 10.1002/pmh.1290

\title{
Commentary
}

\section{Emotion regulation in borderline personality disorder: The role of self-criticism, shame, and self-compassion}

\section{(Commentary on emotional processing in a ten-session general psychiatric treatment for borderline personality disorder: A case study)}

RICKS WARREN, Department of Psychiatry, University of Michigan, 4250 Plymouth Road, Ann Arbor, MI 48109-2700, United States

This case study evaluates the efficacy of a 10-session manualized version of general psychiatric management (GPM) with a female patient diagnosed with borderline personality disorder (BPD), severe depressive disorder, and schizotypal traits. The authors hypothesized that increases in adaptive emotional processing would lead to increases in therapeutic alliance and treatment outcome. Hypotheses were not supported, and in fact, 'a general outcome deterioration tendency and an unchanged alliance level was observed' (p. 7). The patient's level of emotional processing remained at the stage characterized by harsh self-criticism and shame. The authors speculate that it may take more than 10 sessions for changes in emotional processing to occur and that the patient may have been in a state where she would have benefited more from containment, support, and structure.
While this case did not demonstrate therapeutic efficacy of the 10-session GPM treatment or gains in emotional processing, it does represent an attempt to explore possible mechanisms of change in the treatment of borderline personality disorder. The elucidation of mechanisms of change could potentially lead to refinement and modification of currently effective treatments and improvements in therapeutic effectiveness.

Difficulties in emotion regulation are characteristic of many forms of psychopathology (Gross \& Munoz, 1995). As BPD is uniquely characterized by difficulties in emotional regulation, improvements in emotional regulation would appear to be a potential core mechanism in effective treatments for BPD. In support of this, Goodman et al. (2014) reported improvement in emotion regulation associated with decreased amygdala activation in BPD 
patients treated with standard 12-month DBT. In addition, Gratz and Gunderson (2006) reported effectiveness of a 14-week acceptance-based emotion regulation group intervention in reducing emotion dysregulation, BPD specific symptoms, and symptoms of depression, anxiety, and stress. A 16-week DBT skills training group resulted in increases in adaptive emotional processing which was associated with decreases in depression symptoms in patients with treatment-resistant major depressive disorder (Feldman, Harley, Kerrigan, Jacobo, \& Fava, 2009).

While anger and hostility may be the most obvious and intense emotions that persons with BPD have difficulty regulating, clinical and recent research findings suggest that shame may be the most central emotion in BPD (Rüsch et al., 2007) and the emotion most linked with anger, impulsivity, nonsuicidal self-injury (NSSI), and chronic suicidality. Shame, however, may be concealed by BPD patients and overshadowed by the expressed anger and hostility that result from core shame. Failure to recognize shame within the therapy relationship may jeopardize treatment success, and therapy procedures to directly target shame are critical (Rüsch et al., 2007).

Self-criticism, the cognitive precursor to shame, may be more prevalent in BPD than in major depression (Southwick, Yehuda, \& Giller, 1995). Hooley, Ho, Slater, and Lockshin (2010) suggest that people who self-injure may choose this method of emotion regulation because of their harsh self-criticism and view of themselves as bad or defective and deserving punishment. The authors found that 'the more people considered themselves to be worthless or inferior, the more willing they were to endure pain' (p. 176). In a subsequent study, Hooley and St. Germain (2013) found that a 5-min cognitive intervention to increase feelings of self-worth resulted in a significant reduction in length of time persons who engage in NSSI were willing to endure physical pain. The authors suggest that cognitively based interventions 'may reduce the extent to which people are inclined to select physically damaging and painful methods to cope with emotional distress' (p. 303). They also indicate that targeting self-criticism is important 'because criticism challenges brain areas involved in emotion regulation' (p. 303).

A promising emotion regulation strategy that directly addresses self-criticism is self-compassion, which consists of self-kindness, common humanity, and mindfulness (Neff, 2003). Self-compassion is associated with less anxiety, depression, loneliness, and self-criticism and greater emotional coping skills, resilience, feelings of social connectedness, and healthier interpersonal relationships. Recent studies have shown that self-compassion is an effective emotion regulation strategy. In response to negative mood induction procedures with college students with major depressive disorder, self-compassion was effective in down regulating depressed mood, particularly for more severely depressed mood (Diedrich, Grant, Hofmann, Hiller, \& Berking, 2014). Self-compassion was found to be an effective emotion regulation strategy for women experiencing shame due to infertility-related stress (Galhardo, Cunha, Pinto-Gouveia, \& Matos, 2013). In a sample of substance abusing adolescents seeking treatment for substance abuse, self-compassion mediated the relationship between childhood maltreatment and emotion regulation (Vetesse, Dyer, Li, \& Wekerle, 2011). Self-compassion also appears to provide an effective emotion regulation strategy in addressing shame in the treatment of eating disorders (Kelly, Carter, \& Borairi, 2013).

In summary, the case study illustrates an example of current efforts to identify mechanisms contributing to therapeutic change in treatments for borderline personality disorder. Improvement in emotional regulation appears to be a likely candidate. Accumulating evidence suggest that self-compassion may be an effective emotion regulation strategy, particularly suited for targeting self-criticism, shame, and feelings of worthlessness that likely lead to anger, hostility, and self-harm. Future trials of DBT and other evidence-based intervention that measure emotion regulation, 
self-criticism, shame, beliefs about self-worth, and self-compassion could lead to further elucidation of mechanisms of change and improvements in treatment for BPD.

\section{References}

Diedrich, A., Grant, M., Hofmann, S. G., Hiller, W., \& Berking, M. (2014). Self-compassion as an emotion regulation strategy in major depressive disorder. Behaviour Research and Therapy, 58, 43-51. Doi: 10.1016/j. brat.2014.05.006

Feldman, G., Harley, R., Kerrigan, M., Jacobo, M., \& Fava, M. (2009). Change in emotional processing during a dialectical behavior therapy-based skills group for major depressive disorder. Behaviour Research and Therapy, 47, 316-321. Doi: 10.1016/j.brat.2009.01.005

Galhardo, A., Cunha, M., Pinto-Gouveia, J., \& Matos, M. (2013). The mediator role of emotion regulation processes on infertility-related stress. Journal of Clinical Psychology in Medical Settings, 20, 497-507. Doi: 10.1007/ s10880-013-9370-3

Goodman, M., Carpenter, D., Tang, C. Y., Goldstein, K. E., Avedon, J., Fernandez, N., \& Hazlett, E. A. (2014). Dialectical behavior therapy alters emotion regulation and amygdala activity in patients with borderline personality disorder. Journal of Psychiatric Research, 57, 108-116. Doi: 10.106/j.psychires.2014-06-020

Gratz, K. L., \& Gunderson, J. G. (2006). Preliminary data on an acceptance-based emotion regulation group intervention for deliberate self-harm among women with borderline personality disorder. Behavior Therapy, 37, 25-35.

Gross, J. J., \& Munoz, R. F. (1995). Emotion regulation and mental health. Clinical Psychology: Science and Practice, 2, 151-164.
Hooley, J. M., Ho, D. T., Slater, J., \& Lockshin, A. (2010). Pain perception and nonsuicidal self-injury: a laboratory investigation. Personality Disorders: Theory, Research, and Treatment, 3, 170-179. Doi: 10.1037/a0020106

Hooley, J. M., \& St. Germain, S. A. (2013). Nonsuicidal selfinjury, pain, and self-criticism: does changing self-worth change pain endurance in people who engage in self-injury? Clinical Psychological Science, 2, 297-305. Doi: 10.1177/2167702613509372

Kelly, A. C., Carter, J. C., \& Borairi, S. (2013). Are improvements in shame and self-compassion early in eating disorders treatment associated with better patient outcomes? International Journal of Eating Disorders, 47, 54-64.

Neff, K. D. (2003). The development and validation of a scale to measure self-compassion. Self and Identity, 2, 223-250. Doi: 10.1080/15298860390209035

Rüsch, N., Lieb, K., Göttler, I., Hermann, C., Schramm, E., Richter, H., \& Bohus, M. (2007). Shame and implicit self-concept in women with borderline personality disorder. American Journal of Psychiatry, 164, 500-508.

Southwick, S. M., Yehuda, R., \& Giller, E. L. (1995). Psychological dimensions of depression in borderline personality disorder. American Journal of Psychiatry, 152, 789-791.

Vetesse, L. C., Dyer, C. E., Li, W. L., \& Wekerle, C. (2011). Does self-compassion mitigate the association between childhood maltreatment and later emotion regulation difficulties? A preliminary investigation. International Journal of Mental Health and Addiction, 9, 480-489. Doi: 10.1007/ s11469-011-9340-7

Address correspondence to: Ricks Warren, University of Michigan Department of Psychiatry, 4250 Plymouth Road Ann Arbor, MI 48109-2700, USA. Email: ricksw@med.umich.edu 